\title{
Принципы обратной моноимпульсной радилокации в задачах построения помехоустойчивых и живучих систем самонаведения
}

Рассмотрены принципы построения обратных моноимпульсных систем, обеспечивающих повышенную помехоустойчивость за счёт излучения суммарным и разностными каналами, а приёма одним суммарным каналом. Показано, что обратная схема построения моноимпульсного пеленгатора даёт возможность формировать приёмный суммарный канал в виде весовой суммы нескольких отдельных каналов, что обеспечивает подавление помех в главном максимуме диаграммы направленности его антенны. С другой стороны, приём одним каналом даёт возможность выделения пеленга цели относительно излучающего элемента пассивными радиолокационными средствами, не совмещенными с передатчиком.

Ключевые слова: моноимпульсная система, суммарный канал, разностный канал, зенитный ракетный комплекс, зенитная ракетная система.

\section{Введение}

В полуактивных или активных радиолокационных системах самонаведения зенитных ракетных комплексов (ЗРК) и систем (ЗРС) средней и большой дальности действия величина ошибок измерения координат и параметров цели уменьшается с сокращением расстояния между ракетой и целью. Независимо от принципа построения системы самонаведения - система самонаведения в чистом виде или комбинация системы самонаведения и командной радиолинии управления (сопровождение цели через ракету) - ключевым информационным элементом зенитной ракеты является радиолокационная головка самонаведения (РГС).

В РГС, как правило, используются либо классические моноимпульсные схемы построения радиолокационных устройств $[1,2,3]$, либо схемы со скрытым сканированием (моноконические пеленгаторы) [4], формируемые из моноимпульсных устройств за счёт уплотнения пространственных каналов. Главной особенностью моноимпульсных систем является способность путём сравнения амплитуд или фаз сигналов, принятых с помощью специальной антенны, почти мгновенно определять угловые рассогласования объекта локации (цели) относительно равносигнального направления (РСH). Благодаря принципу многоканальности антенны в режиме приёма возможно мгновенное выделение угловых рассогласований, принятых пространственными каналами с отличающимися характеристиками, основанное на разности фаз или амплитуд. Такой принцип построения обеспечивает нечувствительность моноимпульсного радиолокатора к различным помехам, уводящим по направлению, если, конечно, они не вынесены за пределы геометрии цели.

Однако достоинство моноимпульсных радиолокационных устройств, заключающееся в мгновенности измерения угловых рассогласований, сразу же превращается в недостаток, как только речь идёт о помехах, которые, будучи поставлены из точек, вынесенных за геометрические размеры цели, искажают амплитудно-фазовое распределение электромагнитного поля на апертуре антенны. Такими помехами могут быть точечные по пространству помехи, спектральные составляющие которых полностью совпадают со спектром отражённого от цели сигнала, т. е. имитирующие помехи с полностью совпадающими параметрами (дальность, частота Доплера) или шумовые помехи (прямошумовая или модулированная).

Известные методы подавления помех [5], применяемые в радиолокационных устройствах с использованием дополнительных приёмных каналов, обеспечивают эффективную компенсацию шумовых помех по боковым лепесткам диаграмм направленности (ДН) антенн [6, 7]. Методы компенсации, основанные на использовании выборочных пространственно-временных оценок пространственной корреляционной матрицы, не обеспечивают подавления мощных имитирующих помех ввиду их сильной корреляции с полезным сигналом. Знание направления на источник помехи, действующий по боковым лепесткам, также не даёт возможности её скомпенсиро- 
вать, т. к. значения комплексных характеристик направленности (XH) боковых лепестков на практике трудно измерить. Кроме того, комплексные ХН всё время меняются в зависимости от внешних условий.

При компенсации помехи в основном лепестке результирующая комплексная ХН пространственного канала (суммарного, разностного или парциального) представляет собой взвешенную сумму комплексных ХН исходного и компенсационного каналов. При использовании для компенсации помех одного и того же канала во всех исходных пространственных каналах моноимпульсного пеленгатора (МИП) положение фазовых центров результирующих каналов изменяется так, что применение обычных методов моноимпульсной радиолокации приводит к искажениям пеленгационной характеристики (ПХ), так что теряется возможность измерения угловых рассогласований. В этом случае ПХ МИП теряет форму нечётной функции.

Цель данной работы - обосновать необходимость применения в ЗРК и ЗРС моноимпульсных радиолокационных систем обратного типа, которое позволит существенно повысить их помехоустойчивость и живучесть в условиях применения противорадиолокационных ракет.

\section{Принципы построения и свойства обратных моноимпульсных систем}

В классических моноимпульсных системах угловая информация извлекается из мгновенных разностей амплитуд или фаз волн принятых сигналов, отражённых от объекта локации. Для этого формируются либо парциальные, либо суммарные и разностные диаграммы направленности (ДН) антенны, позволяющие после оптимальной обработки сигналов в приёмных трактах сравнить отклики оптимальных фильтров на предмет разностей фаз или амплитуд.

На современном этапе развития радиолокационной техники широко применяются схемы многоканальных радиолокационных систем (РЛС) с цифровыми приёмо-передающими модулями (ППМ), состоящими из аналогового приёмопередатчика, цифроаналогового преобразователя (ЦАП) и аналого-цифрового преобразователя (АЦП) (рис. 1). В цифровой части методами прямого цифрового синтеза либо с помощью генерации числовых последовательностей заданной частоты формируются цифровые сигналы, преобразуемые в аналоговую форму с помощью ЦАП. Аналоговый сигнал усиливается и преобразуется на несущую частоту с помощью усилительно-преобразовательной цепочки передатчика (ПРД), а принятый сигнал усиливается и преобразуется на промежуточную частоту с помощью супергетеродинного приёмника (ПРМ). Для формирования гетеродинных и опорных частот используется блок задающих, гетеродинных и опорных сигналов (БЗГОС). Развязка между приёмником и передатчиком выполняется с помощью циркулятора (Ц) совместно со специальными устройствами коммутации из состава ПРД и ПРМ. На промежуточной частоте принятый сигнал преобразуется в цифровую форму с помощью АЦП и поступает в цифровую часть для дальнейшей обработки. При наличии многоканальной РЛС с цифровым формированием и обработкой сигнала можно реализовать другой принцип построения моноимпульсной системы, которую назовем обратной.

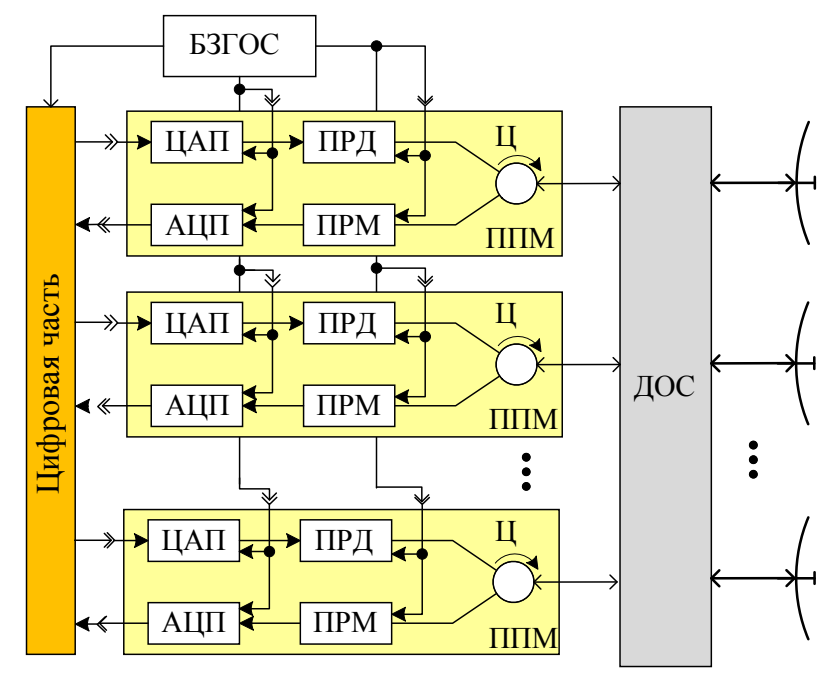

Рис. 1. Обобщённая структурная схема цифровой РЛС

\section{Обобщённая моноимпульсная система обратного типа}

Под обратной моноимпульсной системой будем понимать такую радиолокационную систему, в которой сигнал излучается с помощью 
суммарной, разностных или парциальных (секторных) ДН, а принимается только суммарной ДН. Допустим, что система состоит из передающих каналов $(r=1,2, \ldots R)$, каждый из которых через диаграммообразующую схему (ДОС) формирует при излучении комплексную $\mathrm{XH} \dot{G}_{r}(\varepsilon, \beta)=G_{r}(\varepsilon, \beta) \exp \left[\mathrm{j} \varphi_{r}(\varepsilon, \beta)\right]$, где $G_{r}(\varepsilon, \beta)$ - амплитудная $\mathrm{XH} ; \varphi_{r}(\varepsilon, \beta)$ - фазовая $\mathrm{XH}$ (рис. $2)$. Модель узкополосного зондирующего сигнала канала с номером $r$, для которого частотную характеристику антенны можно считать независимой от частоты, представим в виде: $s_{r}(t)=\operatorname{Re}\left\{A_{r} \dot{s}(t-r T) \dot{G}_{r}(\varepsilon, \beta) \exp \left[\mathrm{j}\left(2 \pi f_{r} t+\varphi_{0}\right)\right]\right\}$, где $A_{r}$ - амплитуда излучаемого $r$-м каналом зондирующего сигнала, определяемая мощно- стью передатчика;

$$
\dot{s}(t)=U(t) \exp (\mathrm{j}[2 \pi \Delta f(t) t+\Delta \varphi(t)])-\text { ком - }
$$

плексная огибающая зондирующего сигнала;

$U(t)$ - амплитудная модуляция зондирующего сигнала;

$\Delta f(t)$ - частотная модуляция;

$\Delta \varphi(t)$ - фазовая модуляция;

$T$ - длительность временного интервала зондирования канала с номером $r$ (временной интервал между зондированием разных каналов при временном разделении каналов);

$f_{r}$ - несущая частота канала с номером $r$ при частотном разделении каналов;

$\varphi_{0}$ - начальная фаза.

Сигнал, излученный $r$-м каналом моно-
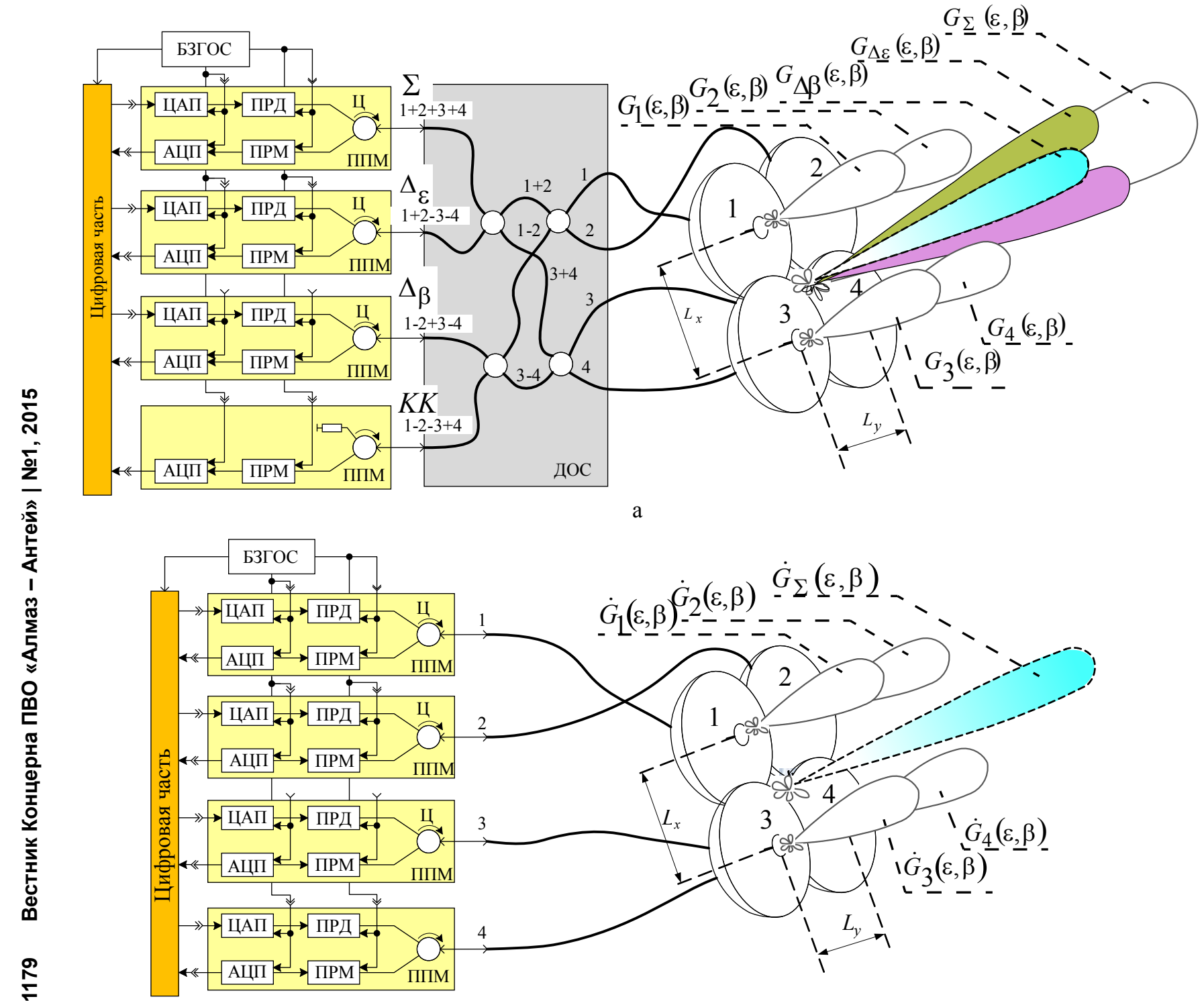

6

Рис. 2. Обобщённые структурные схемы цифровой фазовой обратной моноимпульсной радиолокационной системы: a - с диаграммообразующей суммарно-разностной схемой на передачу и приём; б - с цифровым (программным) формированием суммарной и разностных ДН на передачу 
импульсной антенны, отражается от цели с координатами $\varepsilon_{\text {u }}, \beta_{\text {u }}$ и принимается одновременно $R^{\prime}$ приёмными каналами $\left(r^{\prime}=1,2, \ldots R^{\prime}\right)$, преобразуется в их ППМ на промежуточные частоты, усиливается, затем преобразуется в цифровую форму в АЦП, переносится на нулевую частоту с помощью квадратурного цифрового преобразователя частоты вниз и подвергается фильтрации-децимации с шагом дискретизации $\Delta t$, после чего его можно представить в комплексном виде:

$$
\begin{aligned}
\dot{s}_{r, r^{\prime}}(n)= & A_{\vartheta}(\Delta t n) \sqrt{G_{r}} \mid \dot{G}_{r}\left(\varepsilon_{\text {ц }}, \beta_{\text {ц }}\right) \sqrt{G_{r^{\prime}}} \dot{G}_{r^{\prime}}\left(\varepsilon_{\text {ц }}, \beta_{\text {ц }}\right) \times \\
& \times \dot{s}\left(\Delta t n-r \cdot T-\frac{2 D_{\text {ц }}(\Delta t n)}{c}\right) \times
\end{aligned}
$$

$\times \exp \left(\mathrm{j}\left[\varphi_{r}\left(\varepsilon_{\text {ч }}, \beta_{\text {ч }}\right)-\frac{2 \pi}{\lambda} 2 D_{\text {ч }}(\Delta t n)\right]\right)+\dot{\xi}_{r, r^{\prime}}(n),(1)$

где $A_{э}(t)=\sqrt{\left(P \sigma \lambda^{2}\right) /\left((4 \pi)^{3}\left(D_{\text {u }}(t)\right)^{4}\right)}$-пропорциональная энергии импульса амплитуда, получаемая на выходе фильтра-дециматора;

$P$ - импульсная мощность передатчика;

$G_{r}$ - коэффициент усиления $r$-го канала на передачу;

$G_{r^{\prime}}-$ коэффициент усиления $r^{\prime}$-го канала на приём;

$\sigma-$ эффективная поверхность рассеяния цели;

$D_{\text {ч }}(t)=D_{0}-V t-$ дальность до цели;

$D_{0}$ - дальность до цели на момент начала зондирования каналом с номером 0;

$V$ - радиальная скорость цели (при сближении берётся с отрицательным знаком);

$\dot{\xi}_{r, r}(n)$ - шум приёмника $r^{\prime}$-го канала при зондировании $r$-м каналом.

Если допустить, что амплитуда отражённого сигнала $A_{9}(\mathrm{t})=A_{\ni}$ постоянна за время зондирования (на самом деле она изменяется незначительно), то после оптимальной обработки сигнала (1) с помощью свёртки $\dot{s}_{\mathrm{c} r, r^{\prime}}(n)=\sum_{k=0}^{K-1} \dot{s}_{r, r^{\prime}}(n+k) \dot{g}(k)$ в согласованном фильтре с импульсной характеристикой $\dot{g}(t)=\dot{s}^{*}(t) \exp (-\mathrm{j}[2 \pi / \lambda] 2 \widehat{V} t)$ длительностью $K$ при $n_{\text {ц }}=\left[r \cdot(T / \Delta t)+\left(2 D_{0} / c \Delta t\right)\right]$ получим отклики согласованного фильтра:

$$
\dot{s}_{\mathrm{c} r, r^{\prime}}\left(n_{\text {ц }}\right)=A_{\ni} \sqrt{G_{r}}\left|\dot{G}_{r}\left(\varepsilon_{\text {ц }}, \beta_{\text {ц }}\right)\right| \times
$$

$$
\begin{gathered}
\times \sqrt{G_{r^{\prime}}} \dot{G}_{r^{\prime}}\left(\varepsilon_{\text {ц }}, \beta_{\text {ц }}\right) \exp \left[\mathrm{j} \varphi_{r}\left(\varepsilon_{\text {ц }}, \beta_{\text {ц }}\right)\right] \times \\
\times \exp \left(\mathrm{j} \frac{4 \pi}{\lambda}\left[-D_{0}+(V-\widehat{V}) r \cdot T+\frac{2(V-\widehat{V}) D_{0}}{c}\right]\right) \times \\
\times \sum_{k=0}^{K-1} \dot{s}(k) \dot{g}(k) \exp (\mathrm{j}[(2 \pi / \lambda) 2(V-\widehat{V}) \Delta t k])+ \\
+\dot{\xi}_{\mathrm{c} r, r^{\prime}}\left(n_{\text {ц }}\right),
\end{gathered}
$$

где $\widehat{V}$ - известная оценка радиальной скорости цели;

$$
\dot{\xi}_{\mathrm{c} r, r^{\prime}}\left(n_{\text {щ }}\right)=\sum_{k=0}^{K-1} \dot{\xi}_{r, r^{\prime}}\left(n_{\text {щ }}+k\right) \dot{g}(k) \text { - значение от- }
$$

счёта собственного шума на выходе согласованного фильтра в момент $n=n_{\text {щ }}$.

В ведём комплексную функ цию рассогласования по скорости $\dot{\rho}(\hat{V})=\sum_{k=0}^{K-1} \dot{s}(k) \dot{g}(k) \exp \left(\mathrm{j}\left[\frac{2 \pi}{\lambda} 2(V-\hat{V}) \Delta t k\right]\right)$, которая при $\widehat{V}=V$ равна величине, пропорциональной энергии сигнала, т. е. $\dot{\rho}(\widehat{V}=V)=$ Э.

$$
\text { Обозначив } \frac{2 \pi}{\lambda} 2 D_{0}\left(-1+\frac{2(V-\hat{V})}{c}\right)=\psi_{0} \text {, }
$$

перепишем (2) и получим отклик согласованного фильтра канала $r^{\prime}$ на сигнал, излучённый каналом $r^{\prime}$ :

$$
\begin{aligned}
& \dot{s}_{\mathrm{c} r, r^{\prime}}\left(n_{\text {ц }}\right)=A_{\ni} G_{r}\left|\dot{G}_{r}\left(\varepsilon_{\text {щ }}, \beta_{\text {ц }}\right)\right| \exp \left[\mathrm{j} \varphi_{r}\left(\varepsilon_{\text {ц }}, \beta_{\text {ц }}\right)\right] \times \\
& \times G_{r^{\prime}} \dot{G}_{r^{\prime}}\left(\varepsilon_{\text {ц }}, \beta_{\text {ц }}\right) \exp \left(\mathrm{j} \psi_{0}\right) \exp \left(\mathrm{j} \frac{2 \pi}{\lambda} 2(V-\widehat{V}) r \cdot T\right) \times \\
& \times \dot{\rho}(\widehat{V})+\dot{\xi}_{\mathrm{c} r, r^{\prime}}\left(n_{\mathrm{u}}\right) .
\end{aligned}
$$

Зная радиальную скорость с ошибкой $\Delta V=V_{r}-\widehat{V}_{r}$, при временно́ разделении зондирований каналов можно скомпенсировать изменение дальности цели с точностью до фазового сдвига $\Delta \varphi=(2 \pi / \lambda) 2(V-\hat{V}) \cdot T$ [8]. Чем точнее измерена радиальная скорость, тем лучше компенсация радиальной скорости цели, тем точнее будут оценки пеленгов.

Введём ещё раз некий комплексный коэффициент

$$
\dot{a}_{r}=\exp \left(\mathrm{j} \psi_{0}\right) \exp \left(\mathrm{j} \frac{2 \pi}{\lambda} 2(V-\widehat{V}) r \cdot T\right) \dot{\rho}(\widehat{V})
$$

и перепишем (3) как

$$
\begin{aligned}
\dot{s}_{\mathrm{c} r, r^{\prime}}\left(n_{\text {ц }}\right)= & \dot{a}_{r} A_{\ni} \sqrt{G_{r}} \mid \dot{G}_{r}\left(\varepsilon_{\text {ц }}, \beta_{\text {ц }}\right) \exp \left[\mathrm{j} \varphi_{r}\left(\varepsilon_{\text {ц }}, \beta_{\text {ц }}\right)\right] \times \\
& \times \sqrt{G_{r^{\prime}}} \dot{G}_{r^{\prime}}\left(\varepsilon_{\text {ц }}, \beta_{\text {ц }}\right)+\dot{\xi}_{\mathrm{c} r, r^{\prime}}\left(n_{\text {ц }}\right) .
\end{aligned}
$$

Выражение (4) является фундаментальной записью, доказывающей наличие угловой 
информации о цели в сигнале, закладываемой при передаче (множитель комплексной ХН $\left.\sqrt{G_{r}}\left|\dot{G}_{r}\left(\varepsilon_{\text {ц }}, \beta_{\text {ц }}\right)\right| \exp \left[\mathrm{j} \varphi_{r}\left(\varepsilon_{\text {ц }}, \beta_{\text {ц }}\right)\right]\right)$ и при приёме (множитель $\sqrt{G_{r^{\prime}}} \dot{G}_{r^{\prime}}\left(\varepsilon_{\text {ц }}, \beta_{\text {ц }}\right)$ ). При этом во многих практических задачах на довольно коротких интервалах зондирования можно считать

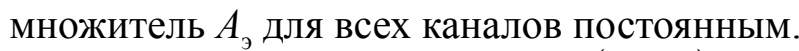

По сути, приёмные ХН $\dot{G}_{r^{\prime}}\left(\varepsilon_{\text {ц }}, \beta_{\text {ц }}\right)$ могут быть произвольными. Главным требованием является их независимость от номеров передающих каналов $r$. Это означает, что независимо от принципа разделения каналов (временно́е или частотное) ХН приёмных каналов одинаковы в моменты зондирования или на частотах зондирования с номером $r$.

Если сигнал может быть принят совокупностью нескольких приёмных каналов с ХН $\dot{G}_{r^{\prime}}\left(\varepsilon_{\text {u }}, \beta_{\text {ц }}\right)$, то затем может быть выполнена весовая обработка сигналов вида

$$
\begin{gathered}
\dot{s}_{\mathrm{c} r}\left(n_{\text {ц }}\right)=\sum_{r^{\prime}=1}^{R} \dot{b}_{r^{\prime}} \dot{s}_{\mathrm{c} r, r^{\prime}}\left(n_{\mathrm{L}}\right)= \\
=\dot{a}_{r} A_{\ni} \sqrt{G_{r}}\left|\dot{G}_{r}\left(\varepsilon_{\text {ц }}, \beta_{\text {ц }}\right)\right| \exp \left[\mathrm{j} \varphi_{r}\left(\varepsilon_{\text {ц }}, \beta_{\text {ц }}\right)\right] \times \\
\times \sum_{r^{\prime}=1}^{R} \dot{b}_{r^{\prime}} \sqrt{G_{r^{\prime}}} \dot{G}_{r^{\prime}}\left(\varepsilon_{\text {ц }}, \beta_{\text {ц }}\right)+\sum_{r^{\prime}=1}^{R} \dot{b}_{r^{\prime}} \dot{\xi}_{\mathrm{c} r, r^{\prime}}\left(n_{\text {ц }}\right)
\end{gathered}
$$

с весовыми коэффициентами $b_{r^{\prime}}$ так, чтобы для каждого канала зондирования с номером сформировалась при приёме результирующая (суммарная) ХН вида

$$
\dot{G}_{\Sigma r}(\varepsilon, \beta)=\sum_{r^{\prime}=1}^{R} \dot{b}_{r^{\prime}} \sqrt{G_{r^{\prime}}} \dot{G}_{r^{\prime}}(\varepsilon, \beta),
$$

обеспечивающая нуль (провал) в направлении источника помех $\varepsilon_{\text {п }}, \beta_{\text {п }}$ [5-7]. Свойство разделения ХН на передачу и на приём даёт возможность подавлять помеху в режиме приёма, сохраняя угловую информацию цели в сигналах $\dot{s}_{\mathrm{c} r}\left(n_{\text {u }}\right)$ с номерами $r$, излученных каналами $r=1,2,3 \ldots R$.

Ещё одной немаловажной особенностью обратной моноимпульсной системы является возможность использования разнесённых в пространстве пунктов передачи и приёма сигналов. Приёмный канал с ХН $\dot{G}_{r^{\prime}}(\varepsilon, \beta)$ или их совокупностью может быть реализован в другой точке приёма, не совмещённой с передающей частью радиолокационной системы. Например, в случае с ЗРК или ЗРС передающие каналы могут быть выполнены в РГС ракеты, а приёмные каналы - на радиолокационной станции наведения, работающей в пассивном режиме приёма сигналов РГС, переотражённых от цели. Таким образом, можно закладывать информацию об угловых отклонениях цели от равносигнального направления РГС в сигнале, принятом на наземном пункте наведения, обеспечивая живучесть станции, работающей в пассивном режиме.

\section{Примеры построения фазовых}

\section{и амплитудных суммарно-разностных} моноимпульсных систем обратного типа

В настоящее время в современной радиолокационной технике, осуществляющей сопровождение целей, широкое распространение получили фазовые и амплитудные суммарноразностные системы. Рассмотрим несколько вариантов обратных моноимпульсных суммарно-разностных систем, отличающихся принципами построения антенн и реализации процедур вычисления пеленга. Под процедурами вычисления пеленга будем понимать вычисление аддитивного отношения сигналов, используемых в суммарно-разностных системах $[1,2]$. Фазовая обратная суммарно-разностная моноимпульсная система

Главная особенность такой системы заключается в наличии антенны, комплексные нормированные ХН частей которой $\dot{G}_{1}(\varepsilon, \beta), \dot{G}_{2}(\varepsilon, \beta)$, $\dot{G}_{3}(\varepsilon, \beta)$ и $\dot{G}_{4}(\varepsilon, \beta)$ можно представить как

$$
\begin{gathered}
\dot{G}_{1}(\varepsilon, \beta)=G_{0}(\varepsilon, \beta) ; \\
\dot{G}_{2}(\varepsilon, \beta)=G_{0}(\varepsilon, \beta) \exp \left(\mathrm{j}[2 \pi / \lambda] L_{y} \cos \varepsilon \sin \beta\right) ; \\
\dot{G}_{3}(\varepsilon, \beta)=G_{0}(\varepsilon, \beta) \times \\
\times \exp \left(\mathrm{j}[2 \pi / \lambda]\left[L_{x} \sin \varepsilon+L_{y} \cos \varepsilon \sin \beta\right]\right) ; \\
\dot{G}_{4}(\varepsilon, \beta)=G_{0}(\varepsilon, \beta) \exp \left(\mathrm{j}[2 \pi / \lambda] L_{x} \sin \varepsilon\right),
\end{gathered}
$$

где $G_{0}(\varepsilon, \beta)$ - амплитудная ХН одной антенны (сектора антенны);

$L_{x}, L_{y}$ - расстояния между фазовыми центрами антенн в вертикальной (угломестной) и горизонтальной (азимутальной) плоскостях [8].

Структурные схемы вариантов фазовой суммарно-разностной системы приведены на рис. 2. На рис. 2а индексы $r$ - номера секторов антенны, из которых с помощью ДОС формируются суммарный $(\Sigma)$, разностный угломестный $\left(\Delta_{\varepsilon}\right)$, разностный азимутальный $\left(\Delta_{\beta}\right)$ и квадрупольный (КК) каналы при зондировании с $\mathrm{XH} \sqrt{G_{\Sigma}}\left|\dot{G}_{\Sigma}(\varepsilon, \beta), \sqrt{G_{\Delta_{\varepsilon}}}\right| \dot{G}_{\Delta_{\varepsilon}}(\varepsilon, \beta), \sqrt{G_{\Delta_{\beta}}}\left|\dot{G}_{\Delta_{\beta}}(\varepsilon, \beta)\right|$ и $\sqrt{G_{\text {Кк }}} \mid \dot{G}_{\text {кК }}(\varepsilon, \beta)$. Индексы $r=1,2,3,4$ обознача- 
ют те же сектора антенны, с помощью которых формируются приёмные суммарный, разностные и квадрупольный каналы с ХН $\sqrt{G_{\Sigma^{\prime}}}\left|\dot{G}_{\Sigma^{\prime}}(\varepsilon, \beta)\right|, \quad \sqrt{G_{\Delta_{\varepsilon}^{\prime}}}\left|\dot{G}_{\Delta_{\varepsilon}^{\prime}}(\varepsilon, \beta), \quad \sqrt{G_{\Delta_{\beta}^{\prime}}}\right| \dot{G}_{\Delta_{\beta}^{\prime}}(\varepsilon, \beta)$ $\sqrt{G_{\mathrm{KK}^{\prime}}}\left|\dot{G}_{\text {КК' }}(\varepsilon, \beta)\right|$. Тогда в сигнале (4) вместо индексов $r$ и $r^{\prime}$ будут использованы индексы $\Sigma, \Delta_{\varepsilon}$, $\Delta_{\beta}$, КК, т. е., например, $\dot{s}_{\mathrm{c} \Sigma, \Delta_{\varepsilon}^{\prime}}(n)$ - отражённый от цели обрабатываемый разностным угломестным каналом на приём $\Delta_{\varepsilon}^{\prime}$ сигнал, излучённый суммарным каналом $\Sigma$ на передачу. Для защиты от помех в качестве компенсационного канала, например, может использоваться квадрупольный канал. В этом случае известными методами адаптивной обработки пар сигналов $\dot{s}_{\mathrm{c} \Sigma, \Sigma^{\prime}}(n)$ и $\dot{s}_{\mathrm{c} \Sigma, \text { КК' }}(n), \dot{s}_{\mathrm{c} \Delta_{\varepsilon}, \Sigma^{\prime}}(n)$ и $\dot{s}_{\mathrm{c} \Delta_{\varepsilon}, \text { КК }}(n), \dot{s}_{\mathrm{c} \Delta_{\varepsilon}, \Sigma^{\prime}}(n)$ и $\dot{s}_{\mathrm{c} \Delta_{\varepsilon}, \text { кК' }}(n)$ подбираются такие весовые коэффициенты для квадрупольных каналов, которые обеспечивают вычитание помехи при операциях:

$$
\begin{aligned}
\dot{s}_{\mathrm{p} \Sigma, \Sigma^{\prime}}(n) & =\dot{s}_{\mathrm{c} \Sigma, \Sigma^{\prime}}(n)-\dot{b}_{\Sigma} \dot{s}_{\mathrm{c} \Sigma, \mathrm{KK}}(n) ; \\
\dot{s}_{\mathrm{p} \Delta_{\varepsilon}, \Sigma^{\prime}}(n) & =\dot{s}_{\mathrm{c} \Delta_{\varepsilon}, \Sigma^{\prime}}(n)-\dot{b}_{\varepsilon} \dot{s}_{\mathrm{c} \Delta_{\varepsilon}, \mathrm{KK}}(n) ; \\
\dot{s}_{\mathrm{p} \Delta_{\beta}, \Sigma^{\prime}}(n) & =\dot{s}_{\mathrm{c} \Delta_{\beta}, \Sigma^{\prime}}(n)-\dot{b}_{\varepsilon} \dot{s}_{\mathrm{c} \Delta_{\beta}, K K^{\prime}}(n) .
\end{aligned}
$$

Если обозначить результирующую ХН как $\dot{G}_{p \Sigma^{\prime}}(\varepsilon, \beta)=\sqrt{G_{\Sigma^{\prime}}} \mid \dot{G}_{\Sigma^{\prime}}(\varepsilon, \beta)-\dot{b}_{\Sigma} \sqrt{G_{\mathrm{KK}^{\prime}}} \dot{G}_{\mathrm{KK}^{\prime}}(\varepsilon, \beta)$, то зондирующие сигналы суммарного и разностных зондирующих каналов, принятые результирующим суммарным приёмным каналом, можно представить в виде:

$$
\begin{aligned}
& \dot{s}_{\mathrm{p} \Sigma, \Sigma^{\prime}}(n)=\dot{a}_{\Sigma} A_{\ni} \sqrt{G_{\Sigma}}\left|\dot{G}_{\Sigma}\left(\varepsilon_{\text {ц }}, \beta_{\text {ц }}\right)\right| \exp \left[\mathrm{j} \varphi_{\Sigma}\left(\varepsilon_{\text {ц }}, \beta_{\text {ц }}\right)\right] \times \\
& \times \dot{G}_{p \Sigma^{\prime}}\left(\varepsilon_{\mathrm{L}}, \beta_{\mathrm{L}}\right)+\dot{\xi}_{\mathrm{c} \Sigma, \Sigma^{\prime}}(n)-\dot{b}_{\Sigma} \dot{\xi}_{\mathrm{c} \Sigma, \mathrm{KK}}(n) ; \\
& \dot{s}_{\mathrm{p} \Delta_{\varepsilon}, \Sigma^{\prime}}(n)=\dot{a}_{\Delta_{\varepsilon}} A_{\ni} \sqrt{G_{\Delta_{\varepsilon}}} \mid \dot{G}_{\Delta_{\varepsilon}}\left(\varepsilon_{\text {ц }}, \beta_{\text {ц }}\right) \times \\
& \times \exp \left[\mathrm{j} \varphi_{\Delta_{\varepsilon}}\left(\varepsilon_{\text {ц }}, \beta_{\text {ц }}\right)\right] \dot{G}_{p \Sigma^{\prime}}\left(\varepsilon_{\text {ц }}, \beta_{\text {ц }}\right)+ \\
& +\dot{\xi}_{\mathrm{c} \Delta_{\varepsilon}, \Sigma^{\prime}}(n)-\dot{b}_{\Sigma} \dot{\xi}_{\mathrm{c} \Delta_{\varepsilon}, \mathrm{KK}}(n) \text {; } \\
& \dot{s}_{\mathrm{p} \Delta_{\beta}, \Sigma^{\prime}}(n)=\left.\dot{a}_{\Delta_{\beta}} A_{\ni} \sqrt{G_{\Delta_{\beta}}}\right|_{\dot{G}_{\Delta_{\beta}}} ^{\dot{s}_{\mathrm{u}}}\left(\varepsilon_{\mathrm{u}}, \beta_{\mathrm{u}}\right) \mid \times \\
& \times \exp \left[\mathrm{j} \varphi_{\Delta_{\beta}}\left(\varepsilon_{\text {ц }}, \beta_{\text {ц }}\right)\right] \dot{G}_{p \Sigma^{\prime}}\left(\varepsilon_{\text {ц }}, \beta_{\text {ц }}\right)+ \\
& +\dot{\xi}_{\mathrm{c} \Delta_{\beta}, \Sigma^{\prime}}(n)-\dot{b}_{\Sigma} \dot{\xi}_{\mathrm{c} \Delta_{\beta}, K K^{\prime}}(n) \text {. }
\end{aligned}
$$

Отношения второго и третьего разностных сигналов к суммарному сигналу дают аддитивные отношения, с помощью которых вычисляются пеленги цели:

$$
\Delta_{\varepsilon}=\operatorname{Im}\left\{\frac{\dot{s}_{\mathrm{p} \Delta_{\varepsilon}, \Sigma^{\prime}}\left(n_{\mathrm{u}}\right)}{\dot{s}_{\mathrm{p} \Sigma, \Sigma^{\prime}}\left(n_{\mathrm{u}}\right)}\right\}=
$$

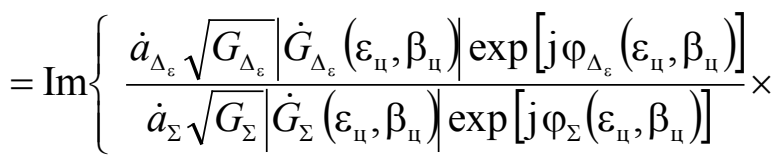

$$
\begin{aligned}
& \left.\times\left(\frac{1+\delta \xi_{\varepsilon}}{1+\delta \xi_{\Sigma}}\right)\right\} \\
& \Delta_{\beta}=\operatorname{Im}\left\{\frac{\dot{s}_{\mathrm{p} \Delta_{\beta}, \Sigma^{\prime}}\left(n_{\mathrm{u}}\right)}{\dot{s}_{\mathrm{p} \Sigma, \Sigma^{\prime}}\left(n_{\mathrm{u}}\right)}\right\}= \\
& =\operatorname{Im}\left\{\frac{\dot{a}_{\Delta_{\beta}} \sqrt{G_{\Delta_{\beta}}}\left|\dot{G}_{\Delta_{\beta}}\left(\varepsilon_{\text {ц }}, \beta_{\text {ц }}\right)\right| \exp \left[\mathrm{j} \varphi_{\Delta_{\beta}}\left(\varepsilon_{\text {ц }}, \beta_{\text {ц }}\right)\right]}{\dot{a}_{\Sigma} \sqrt{G_{\Sigma}}\left|\dot{G}_{\Sigma}\left(\varepsilon_{\text {ц }}, \beta_{\text {ц }}\right)\right| \exp \left[\mathrm{j} \varphi_{\Sigma}\left(\varepsilon_{\text {ц }}, \beta_{\text {ц }}\right)\right]} \times\right. \\
& \left.\times\left(\frac{1+\delta \xi_{\beta}}{1+\delta \xi_{\Sigma}}\right)\right\} \text {. }
\end{aligned}
$$

которые, по сути, определяются отношениями разностных зондирующих ХН к суммарной зондирующей ХН с точностью, определяемой множителями $\left(1+\delta \xi_{\varepsilon} / 1+\delta \xi_{\Sigma}\right)$ и $\left(1+\delta \xi_{\beta} / 1+\delta \xi_{\Sigma}\right)$, где

$$
\begin{aligned}
& \delta \xi_{\varepsilon}=\left(\dot{\xi}_{\mathrm{c} \Delta_{\varepsilon}, \Sigma^{\prime}}\left(n_{\mathrm{u}}\right)-\dot{b}_{\Sigma} \dot{\xi}_{\mathrm{c} \Delta_{\varepsilon}, \mathrm{KK}}\left(n_{\mathrm{u}}\right)\right) /\left(\dot{a}_{\Delta_{\varepsilon}} A_{\ni} \sqrt{G_{\Delta_{\varepsilon}}} \times\right.
\end{aligned}
$$

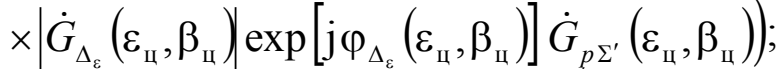

$$
\begin{aligned}
& \delta \xi_{\beta}=\left(\dot{\xi}_{\mathrm{c} \Delta_{\beta}, \Sigma^{\prime}}\left(n_{\mathrm{L}}\right)-\dot{b}_{\Sigma} \dot{\xi}_{\mathrm{c} \Delta_{\beta}, \mathrm{KK}}\left(n_{\mathrm{L}}\right)\right) /\left(\dot{a}_{\Delta_{\beta}} A_{\ni} \sqrt{G_{\Delta_{\beta}}} \times\right.
\end{aligned}
$$

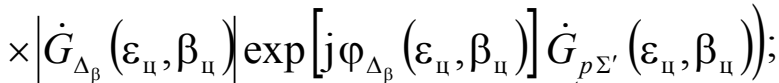

$$
\begin{aligned}
& \delta \xi_{\Sigma}=\left(\dot{\xi}_{\mathrm{c} \Sigma, \Sigma^{\prime}}\left(n_{\mathrm{u}}\right)-\dot{b}_{\Sigma} \dot{\xi}_{\mathrm{c} \Sigma, \mathrm{KK}}\left(n_{\mathrm{u}}\right)\right) /\left(\dot{a}_{\Sigma} A_{\ni} \sqrt{G_{\Sigma}} \times\right.
\end{aligned}
$$

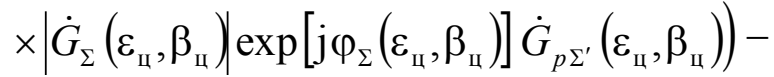
комплексные ошибки, обратные отношениям сигнал/шум в результирующем канале для соответствующих зондирующих каналов. Ошибки пеленга, определяемые шумами, формируются так же, как и в классической моноимпульсной системе, в отличие от которой есть ещё ошибки, определяемые отношениями $\dot{a}_{\Delta_{\varepsilon}} / \dot{a}_{\Sigma}$ и $\dot{a}_{\Delta_{\beta}} / \dot{a}_{\Sigma}$, суть которых будет рассмотрена ниже.

При использовании цифровой схемы формирования каналов (рис. 2б) суммарный и разностные зондирующие каналы можно формировать путём введения фазовых поправок на сигналы отдельных антенн так, чтобы сформировать на передачу суммарную и две разностные ДН, аналогичные тем, которые сформированы с помощью ДОС первой схемы (рис. 2a). При приёме для компенсации помехи целесо- 
образно использовать весовую сумму каналов отдельных секторов:

$$
\dot{G}_{p \Sigma^{\prime}}(\varepsilon, \beta)=\sum_{r^{\prime}=1}^{R} \dot{b}_{r^{\prime}} \sqrt{G_{r^{\prime}}} \dot{G}_{r^{\prime}}(\varepsilon, \beta) .
$$

Нетрудно убедиться, что пеленги цели будут определяться выражениями, аналогичными (7) и (8), т. к. множители $\dot{G}_{p \Sigma^{\prime}}(\varepsilon, \beta)$ в числителе и знаменателе сокращаются. Единственным отличием будет весовая сумма шумов, определяемая как

$$
\begin{gathered}
\delta \xi_{\varepsilon}=\sum_{r^{\prime}=1}^{R} \dot{b}_{\varepsilon r^{\prime}} \dot{\xi}_{\mathrm{c} \Delta_{\varepsilon}, r^{\prime}}\left(n_{\text {ц }}\right) /\left(\dot{a}_{\Delta_{\varepsilon}} A_{\ni} \sqrt{G_{\Delta_{\varepsilon}}} \mid \dot{G}_{\Delta_{\varepsilon}}\left(\varepsilon_{\text {ц }}, \beta_{\text {ц }}\right) \times\right. \\
\left.\times \exp \left[\mathrm{j} \varphi_{\Delta_{\varepsilon}}\left(\varepsilon_{\text {ц }}, \beta_{\text {ц }}\right)\right] \dot{G}_{p \Sigma^{\prime}}\left(\varepsilon_{\text {ц }}, \beta_{\text {ц }}\right)\right) ;
\end{gathered}
$$$$
\delta \xi_{\beta}=\sum_{r^{\prime}=1}^{R} \dot{b}_{\beta r^{\prime}} \dot{\xi}_{\mathrm{c} \Delta_{\beta}, r^{\prime}}\left(n_{\text {ц }}\right) /\left(\dot{a}_{\Delta_{\beta}} A_{\ni} \sqrt{G_{\Delta_{\beta}}}\left|\dot{G}_{\Delta_{\beta}}\left(\varepsilon_{\text {ц }}, \beta_{\text {ц }}\right)\right| \times\right.
$$$$
\left.\times \exp \left[\mathrm{j} \varphi_{\Delta_{\beta}}\left(\varepsilon_{\mathrm{u}}, \beta_{\mathrm{u}}\right)\right] \dot{G}_{p \Sigma^{\prime}}\left(\varepsilon_{\text {u}}, \beta_{\mathrm{u}}\right)\right) ;
$$$$
\delta \xi_{\Sigma}=\sum_{r^{\prime}=1}^{R} \dot{b}_{\Sigma r^{\prime}} \dot{\xi}_{\mathrm{c} \Sigma, r^{\prime}}\left(n_{\text {ц }}\right) /\left(\dot{a}_{\Sigma} A_{\ni} \sqrt{G_{\Sigma}}\left|\dot{G}_{\Sigma}\left(\varepsilon_{\text {ц }}, \beta_{\text {ц }}\right)\right| \times\right.
$$$$
\left.\times \exp \left[\mathrm{j} \varphi_{\Sigma}\left(\varepsilon_{\text {и }}, \beta_{\text {н }}\right)\right] \dot{G}_{p \Sigma^{\prime}}\left(\varepsilon_{\text {и }}, \beta_{\text {н }}\right)\right),
$$

где $\dot{\xi}_{\mathrm{c} \Delta_{\varepsilon}, r^{\prime}}\left(n_{\text {ц }}\right), \dot{\xi}_{\mathrm{c} \Delta_{\beta}, r^{\prime}}\left(n_{\text {ц }}\right), \dot{\xi}_{\mathrm{c} \Sigma, r^{\prime}}\left(n_{\text {ц }}\right)$-шумы приёмников, действующие на выходе согласованного фильтра в процессе излучения или на частотах разностных и суммарного каналов.

Амплитудная обратная суммарно-разностная моноимпульсная система

Для амплитудной системы ХН отдельных каналов будут определяться как

$$
\begin{gathered}
\dot{G}_{0}(\varepsilon, \beta)=G_{0}\left(\varepsilon-\varepsilon_{\text {ск }}, \beta-\beta_{\text {ск }}\right) ; \\
\dot{G}_{1}(\varepsilon)=G_{0}\left(\varepsilon-\varepsilon_{\text {ск }}, \beta+\beta_{\text {ск }}\right) ; \\
\dot{G}_{2}(\varepsilon, \beta)=G_{0}\left(\varepsilon+\varepsilon_{\text {ск }}, \beta+\beta_{\text {ск }}\right) ; \\
\dot{G}_{3}(\varepsilon, \beta)=G_{0}\left(\varepsilon+\varepsilon_{\text {ск }}, \beta-\beta_{\text {ск }}\right),
\end{gathered}
$$

где $G_{0}(\varepsilon, \beta)$ - двухмерная нормированная ХН суммарного канала; $\varepsilon_{\text {ск }}, \beta_{\text {ск }}$ - угол скоса парциальных диаграмм направленности в плоскостях $\varepsilon, \beta$ (рис. 3a).

Принцип формирования суммарного и разностных каналов на передачу идентичен аналогичному принципу, рассмотренному для схем фазовых систем (рис. 2). Следует также отметить, что схемы амплитудных и фазовых обратных моноимпульсных систем могут быть реализованы не только с помощью излучения разностным и суммарным каналами, излучать можно с помощью отдельных секторов антенн (в случае фазовой системы) или парциалов (в случае амплитудной системы). Обработка сигналов в амплитудной системе отличается от фазовой системы тем, что в выражениях (7) и (8) вместо мнимой части комплексного отношения сигналов используется вещественная часть.

В случаях построения амплитудной или фазовой суммарно-разностных систем одним из ключевых является вопрос разделения сигналов суммарного, разностных каналов или разделения сигналов секторов или парциалов (рис. 2a, 3a): если излучает передатчик, подключённый к суммарному каналу, то передатчики, подключенные к разностным и квадрупольному каналам, должны молчать или же излучать на других частотах; если излучает передатчик одного из разностных каналов, то передатчики суммарного и другого разностного и квадрупольного каналов должны молчать.

\section{Принцип временного}

\section{и частотного разделения каналов}

Вопрос о разделении каналов возникает, если исходить из принципа построения обратной моноимпульсной системы. Сам по себе такой принцип построения пеленгатора предусматривает излучение не только суммарным, но и разностными каналами (либо отдельными секторами или парциалами), а приём - одним суммарным каналом или каналом, представляющим весовую сумму сигналов нескольких каналов. Разделить при обработке сигналы суммарного, разностного или парциальных каналов и при этом не исказить физические величины (амплитуду или фазу), несущие информацию об угловом положении цели, можно путём временно́го или частотного разделения каналов. Анализ выражений для зондирующего сигнала предусматривает возможность введения разных частот $f_{r}$ для каждого $r$-го зондирующего канала или временных задержек $T$. Рассмотрим оба варианта разделения каналов. Сразу же напрашивается анализ выражений (7) и (8), в которых за скобкой фигурируют отношения $\dot{a}_{\Delta_{\varepsilon}} / \dot{a}_{\Sigma}$ и $\dot{a}_{\Delta_{\beta}} / \dot{a}_{\Sigma}$. Введём номера разностных и суммарного каналов $r=r_{\varepsilon}, r=r_{\beta}, r=r_{\Sigma}$. Рассмотрим первое отношение $\dot{a}_{\Delta_{\varepsilon}} / \dot{a}_{\Sigma}$. Если подставить в него выражение для функции рассогласования по скорости $\dot{\rho}(\widehat{V})$, то получим: 


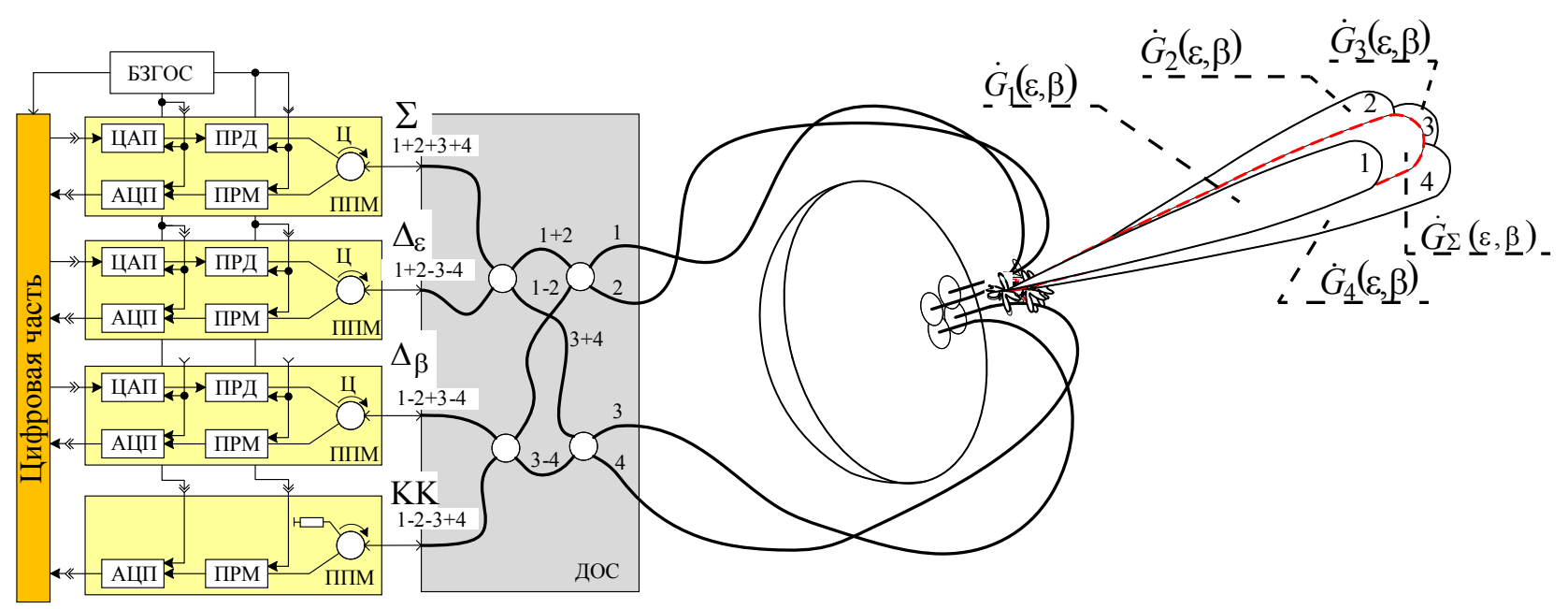

a

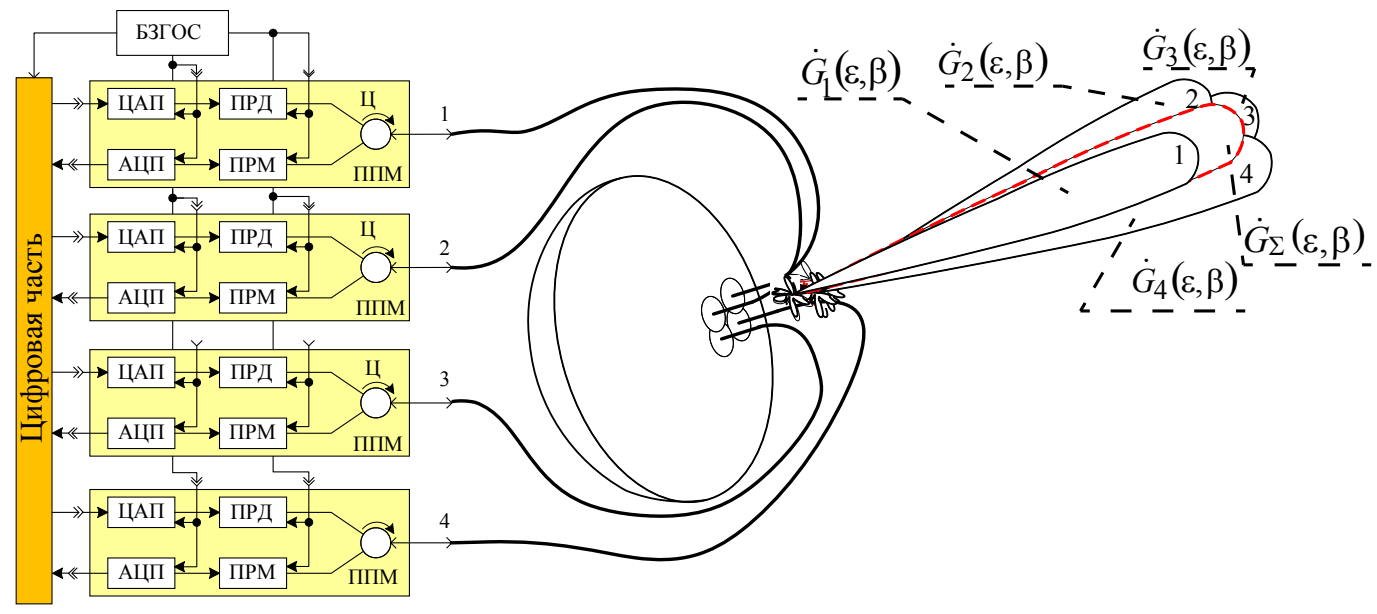

6

Рис. 3. Обобщённые структурные схемы цифровой амплитудной обратной моноимпульсной радиолокационной системы: a - с диаграммообразующей суммарно-разностной схемой на передачу и приём; б - с цифровым (программным) формированием суммарной и разностных ДН на передачу

$\frac{\dot{a}_{\Delta_{\varepsilon}}}{\dot{a}_{\Sigma}}=\frac{\exp \left[\mathrm{j} \psi_{r_{\varepsilon}}+\mathrm{j}\left(\frac{2 \pi}{\lambda_{r_{\varepsilon}}}\right) 2(V-\widehat{V}) r_{\varepsilon} T\right] \dot{\rho}_{r_{\varepsilon}}(\widehat{V})}{\exp \left[\mathrm{j} \psi_{r_{\Sigma}}+\mathrm{j}\left(\frac{2 \pi}{\lambda_{r_{\Sigma}}}\right) 2(V-\widehat{V}) r_{\Sigma} T\right] \dot{\rho}_{r_{\Sigma}}(\widehat{V})} ;(9)$

$\frac{\dot{a}_{\Delta_{\beta}}}{\dot{a}_{\Sigma}}=\frac{\exp \left(\mathrm{j} \psi_{r_{\beta}}+\mathrm{j}\left(\frac{2 \pi}{\lambda_{r_{\beta}}}\right) 2(V-\widehat{V}) r_{\beta} T\right) \dot{\rho}_{\beta}(\widehat{V})}{\exp \left(\mathrm{j} \psi_{r_{r_{\Sigma}}}+\mathrm{j}\left(\frac{2 \pi}{\lambda_{r_{\Sigma}}}\right) 2(V-\widehat{V}) r_{\Sigma} T\right) \dot{\rho}_{\Sigma}(\widehat{V})}$,

где $\psi_{r}=\left(2 \pi f_{r} / c\right)\left(2(V-\hat{V}) 2 D_{0} / c-2 D_{0}\right)$; $\dot{\rho}_{r}(\widehat{V})=\sum_{k=0}^{K-1} \dot{s}(k) \dot{g}(k) \exp \left(\mathrm{j}\left[\frac{2 \pi f_{r}}{c} 2(V-\widehat{V}) \Delta t k\right]\right)$.

В случае временно́го разделения каналов частоты их излучения $f_{r_{2}}, f_{r_{\varepsilon}}, f_{r_{\beta}}$ равны несущей частоте $f_{0}$, а временны́е интервалы зондирования отличаются друг от друга на величину $T$, т. е. $T=r T, r=0,1,2$. В этом случае в выражениях (9) и (10) фазы $\psi_{r_{r_{\varepsilon}}}=\psi_{r_{\mathrm{\beta}}}=\psi_{r_{\Sigma}}$ и множители рассогласования по скорости будут равны $\dot{\rho}_{r_{\varepsilon}}(\widehat{V})=\dot{\rho}_{r_{\beta}}(\widehat{V})=\dot{\rho}_{r_{\bar{z}}}(\widehat{V})$. Отношения (9) и (10) трансформируются к виду:

$$
\begin{aligned}
& \frac{\dot{a}_{\Delta_{\varepsilon}}}{\dot{a}_{\Sigma}}=\exp \left[\mathrm{j}\left(\frac{2 \pi f_{0}}{c}\right) 2(V-\widehat{V})\left(r_{\varepsilon}-r_{\Sigma}\right) \cdot T\right] ; \\
& \frac{\dot{a}_{\Delta_{\beta}}}{\dot{a}_{\Sigma}}=\exp \left[\mathrm{j}\left(\frac{2 \pi f_{0}}{c}\right) 2(V-\widehat{V})\left(r_{\beta}-r_{\Sigma}\right) \cdot T\right],
\end{aligned}
$$

откуда очевидно, что, если не скомпенсировать скорость движения цели $V$, при обработке, т. е. путём ввода комплексно-сопряженной экспоненты с оценкой скорости $\widehat{V}$, то величины (7) и (8) будут иметь угловую ошибку, определяемую ошибкой фазы: 


$$
\begin{aligned}
& \delta \psi_{\varepsilon}=\frac{2 \pi f_{0}}{c} 2(V-\hat{V})\left(r_{\varepsilon}-r_{\Sigma}\right) \cdot T ; \\
& \delta \psi_{\beta}=\frac{2 \pi f_{0}}{c} 2(V-\widehat{V})\left(r_{\beta}-r_{\Sigma}\right) \cdot T .
\end{aligned}
$$

Если, например, несущая частота 15 ГГц, время зондирования одного канала $T=20$ мс, ошибка измерения скорости $1 \mathrm{M} / \mathrm{c}$, a $r_{\varepsilon}=0, r_{\beta}=1, r_{\Sigma}=2$, то $\delta \psi_{\varepsilon}=-1440^{\circ}$, а $\delta \psi_{\beta}=-720^{\circ}$. Отсюда возникает вопрос о необходимости компенсации данных ошибок.

Аналогичная ситуация возникает при частотном разделении каналов. В этом случае $T=0$ и отношения $\dot{a}_{\Delta_{\varepsilon}} / \dot{a}_{\Sigma}, \dot{a}_{\Delta_{\beta}} / \dot{a}_{\Sigma}$, определяющие методическую ошибку, определяются как:

$$
\begin{aligned}
& \dot{a}_{\Delta_{\varepsilon}} / \dot{a}_{\Sigma}=\exp \left[\mathrm{j} 2\left(2 \pi f_{0} / c\right)\left(r_{\varepsilon}-r_{\Sigma}\right) \times\right. \\
& \left.\times\left((V-\widehat{V}) \frac{D_{0}}{c}-D_{0}\right)\right]\left(\dot{\rho}_{r_{\varepsilon}}(\widehat{V}) / \dot{\rho}_{r_{\Sigma}}(\widehat{V})\right) ; \\
& \dot{a}_{\Delta_{\beta}} / \dot{a}_{\Sigma}=\exp \left[\mathrm{j} 2\left(2 \pi f_{0} / c\right)\left(r_{\beta}-r_{\Sigma}\right) \times\right. \\
& \left.\times\left((V-\hat{V}) \frac{D_{0}}{c}-D_{0}\right)\right]\left(\dot{\rho}_{r_{\beta}}(\widehat{V}) / \dot{\rho}_{r_{\Sigma}}(\widehat{V})\right),
\end{aligned}
$$

в которых отношения функций рассогласования $\dot{\rho}_{r}(\widehat{V})$ при согласованности импульсной характеристики с сигналом можно представить в виде:

$$
\begin{gathered}
\frac{\dot{\rho}_{r_{\varepsilon}}(\widehat{V})}{\dot{\rho}_{r_{\Sigma}}(\widehat{V})}= \\
=\frac{\sin \left[K \pi \frac{f_{r_{\varepsilon}}}{c} 2(V-\widehat{V}) \Delta t\right]}{c} \frac{K \sin \left[\pi \frac{f_{\Sigma}}{c} 2(V-\widehat{V}) \Delta t\right]}{K \sin \left[\pi \frac{f_{\varepsilon}}{c} 2(V-\widehat{V}) \Delta t\right] \sin \left[K \pi \frac{f_{r_{\Sigma}}}{c} 2(V-\widehat{V}) \Delta t\right]} \times \\
\times \exp \left[\mathrm{j} \pi \frac{\Delta f}{c}\left(r_{\varepsilon}-r_{\Sigma}\right) 2(V-\widehat{V})(K-1) \Delta t\right] \quad(13) \\
\frac{\dot{\rho}_{r_{\beta}}(\widehat{V})}{\dot{\rho}_{r_{\Sigma}}(\widehat{V})}=
\end{gathered}
$$

$$
\begin{aligned}
= & \frac{\sin \left[K \pi \frac{f_{r_{\beta}}}{c} 2(V-\hat{V}) \Delta t\right]}{K \sin \left[\pi \frac{f_{\beta}}{c} 2(V-\hat{V}) \Delta t\right] \sin \left[\pi \frac{f_{\Sigma}}{c} 2(V-\hat{V}) \Delta t\right]} \times \\
& \times \exp \left[\mathrm{j} \pi \frac{\Delta f}{c}\left(r_{\beta}-r_{\Sigma}\right) 2(V-\hat{V})(K-1) \Delta t\right] .
\end{aligned}
$$

Величины фазовых набегов $K \pi f_{r_{\varepsilon}} 2(V-\hat{V}) \Delta t / c$ и $K \pi f_{r_{\Sigma}} 2(V-\widehat{V}) \Delta t / c$ составляют, как правило, единицы градусов, а фазовые набеги

$$
\begin{aligned}
& \pi \Delta f\left(r_{\varepsilon}-r_{\Sigma}\right) 2(V-\hat{V})(K-1) \Delta t / c \\
& \pi \Delta f\left(r_{\beta}-r_{\Sigma}\right) 2(V-\widehat{V})(K-1) \Delta t / c,
\end{aligned}
$$

обусловленные, разностью частот $\Delta f$, пренебрежимо малы. Величины амплитуд в (13) и (14) близки к единице, и отношения функций рассогласования в (13) и (14) также близки к единице, поэтому при частотном разделении каналов методические ошибки определяются в основном наличием разности частот и текущей дальностью.

Для компенсации паразитных фазовых набегов можно прибегнуть к способам симметрирования. Рассмотрим суть такой компенсации на примере временно́г симметрирования.

Излучим сначала сигнал суммарного зондирующего канала длительностью $T$, который после приёма и адаптивной обработки будет иметь вид:

$\dot{s}_{1 \mathrm{p} \Sigma, \Sigma^{\prime}}(n)=\dot{a}_{1 \Sigma} A_{\ni} \sqrt{G_{\Sigma}} \dot{G}_{\Sigma}\left(\varepsilon_{\text {ц }}, \beta_{\text {ц }}\right) \dot{G}_{p \Sigma^{\prime}}\left(\varepsilon_{\text {u }}, \beta_{\text {ц }}\right)+\dot{\xi}_{1 p \Sigma}(n)$, где $\dot{a}_{1 \Sigma}=\exp \left(\mathrm{j} \psi_{0}\right) \exp [\mathrm{j}(2 \pi / \lambda) 2(V-\hat{V}) r \cdot T] \times$ $\times \dot{\rho}(\widehat{V})=\exp \left(\mathrm{j} \psi_{0}\right) \dot{\rho}(\widehat{V})$, т. к. $r=0$. Затем излучим сигнал разностного угломестного зондирующего канала длительностью $T$, который после приёма и адаптивной (при необходимости) обработки будет иметь вид:

$\dot{s}_{\mathrm{p} \Delta_{\varepsilon}, \Sigma^{\prime}}(n)=\dot{a}_{\Delta_{\varepsilon}} A_{\ni} \sqrt{G_{\Delta_{\varepsilon}}} \dot{G}_{\Delta_{\varepsilon}}\left(\varepsilon_{\text {u }}, \beta_{\text {ц }}\right) \dot{G}_{p \Sigma^{\prime}}\left(\varepsilon_{\text {u }}, \beta_{\text {ц }}\right)+\dot{\xi}_{p \varepsilon}(n)$, где $\dot{a}_{\Delta_{\varepsilon}}=\exp \left(\mathrm{j} \psi_{0}\right) \exp [\mathrm{j}(2 \pi / \lambda) 2(V-\hat{V}) \cdot T] \dot{\rho}(\hat{V})$. Если повторно излучить сигнал суммарным каналом, то после приёма и адаптивной обработки он будет иметь вид:

$\dot{s}_{2 p \Sigma, \Sigma^{\prime}}(n)=\dot{a}_{2 \Sigma} A_{\ni} \sqrt{G_{\Sigma}} \dot{G}_{\Sigma}\left(\varepsilon_{\text {ц }}, \beta_{\text {ц }}\right) \dot{G}_{p \Sigma^{\prime}}\left(\varepsilon_{\text {ц }}, \beta_{\text {и }}\right)+\dot{\xi}_{2 p \Sigma}(n)$, где $\dot{a}_{2 \Sigma}=\exp \left(\mathrm{j} \psi_{0}\right) \exp [\mathrm{j}(2 \pi / \lambda) 2(V-\widehat{V}) 2 \cdot T] \dot{\rho}(\widehat{V})$. Таким образом, видно, что $\dot{a}_{1 \Sigma}=\left|\dot{a}_{1 \Sigma}\right|$, $\dot{a}_{2 \Sigma}=\left|\dot{a}_{2 \Sigma}\right| \exp (\mathrm{j} 2 \Delta \psi)$, где $\Delta \psi=(2 \pi / \lambda) 2(V-\hat{V}) 2 \cdot T$. Расположение векторов сигналов $\dot{s}_{1 \mathrm{p} \Sigma, \Sigma^{\prime}}\left(n_{\text {ц }}\right)$, $\dot{s}_{\mathrm{p} \Delta_{\varepsilon}, \Sigma^{\prime}}\left(n_{\text {ц }}\right)_{\text {и }} \dot{s}_{2 \mathrm{p} \Sigma, \Sigma^{\prime}}\left(n_{\text {ц }}\right)$ показано на рис. 4 . Если сигналы $\dot{s}_{1 \mathrm{p} \Sigma, \Sigma^{\prime}}\left(n_{\text {ц }}\right)$ и $\dot{s}_{2 \mathrm{p} \Sigma, \Sigma^{\prime}}\left(n_{\text {ц }}\right)$ сложить и разделить пополам, то отклики $\dot{s}_{\mathrm{p} \Delta_{\varepsilon}, \Sigma^{\prime}}\left(n_{\text {ц }}\right)$ $\left[\dot{s}_{1 \mathrm{p} \Sigma, \Sigma^{\prime}}\left(n_{\mathrm{u}}\right)+\dot{s}_{2 \mathrm{p} \Sigma, \Sigma^{\prime}}\left(n_{\text {ц }}\right)\right] / 2$ будут совпадать по фазе с точностью до фазовых ошибок, определяемых собственными шумами. Для вычисле- 


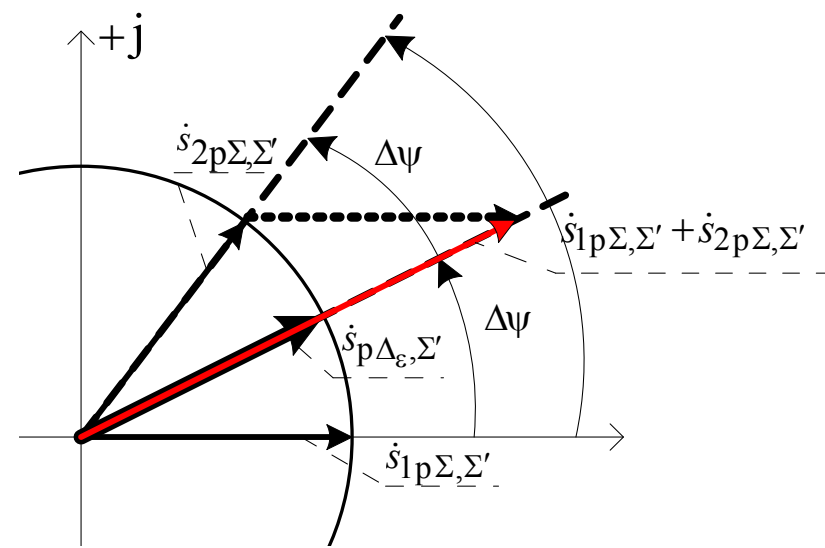

Рис. 4. Комплексная плоскость сигналов компенсировать фазовые сдвиги сигналов, возникающие за счёт временно́го и частотного разделения каналов. Тем не менее, несмотря на некоторые сложности, обратный принцип построения моноимпульсных систем по сравнению с традиционной моноимпульсной системой несет в себе два преимущества:

возможно, принимая сигналы одним лишь каналом, использовать всевозможные алгоритмы компенсации помех, выделяя тем самым на их фоне зондирующие сигналы, и на основе их отношения вычислять пеленги цели; алгоритмы выделения угловой инфор-

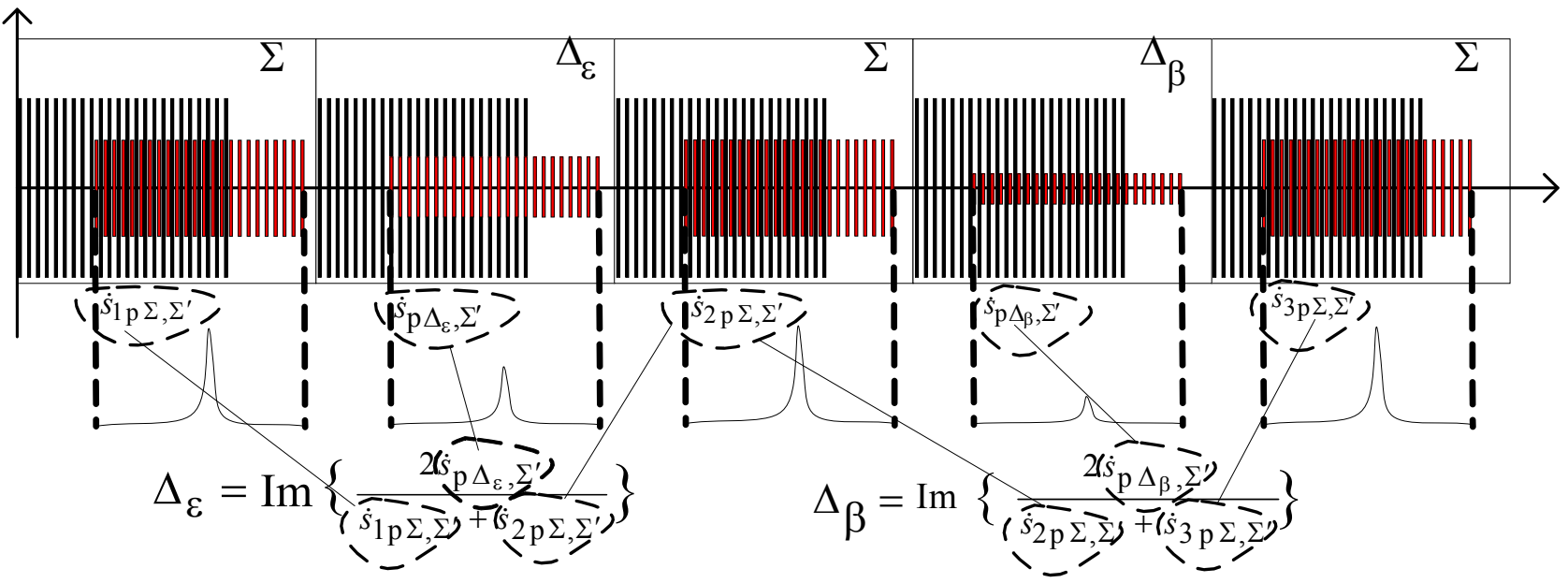

Рис. 5. Симметрично-временное разделение каналов

ния пеленга по другой координате после излучения суммарного сигнала необходимо излучить с помощью разностной азимутальной ДН, а затем снова суммарным каналом. Временна́я диаграмма рассмотренного порядка излучения сигналов показана на рис. 5. Точно так же только при частотном разделении каналов можно распределить частоты разностных каналов относительно суммарного зондирующего канала так, чтобы их частоты располагались симметрично относительно частоты суммарного канала (рис. 6). Это приведет к распределению векторов так же, как это показано на рис. 4.

\section{Заключение}

Моноимпульсная система обратного принципа построения имеет недостаток по сравнению с классической системой: её надо синтезировать путём сложения или вычитания результатов обработки сигналов на разных тактах зондирования или на разных частотах. В этом случае возникает естественная необходимость

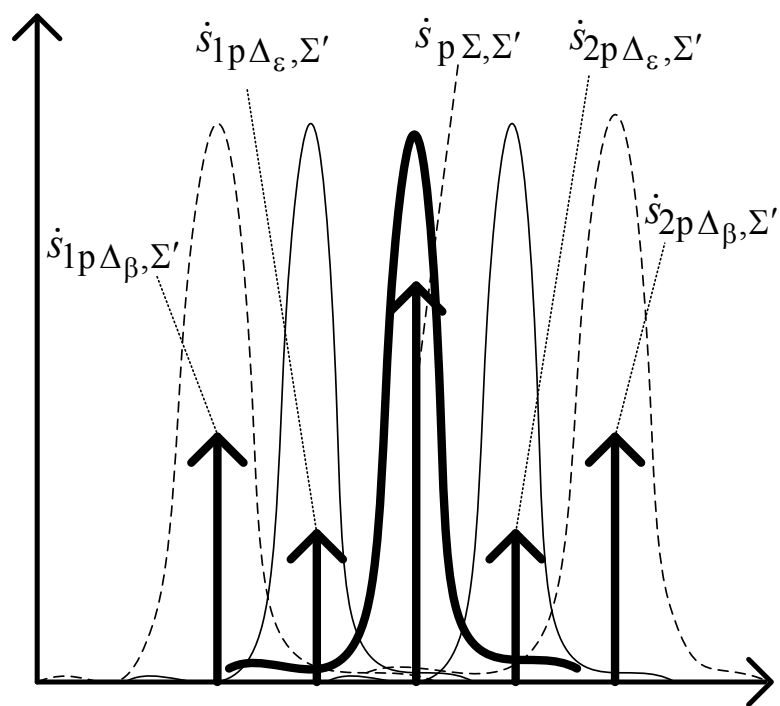

Рис. 6. Симметрично-частотное разделение каналов

мации из сигнала, принятого одним каналом, который модулирован во времени или имеет частотный спектр в соответствии с угловыми рассогласованиями цели, дают возможность строить обратную полуактивную систему наведения, в которой излучающим элементом яв- 
ляется объект управления, а приёмным- станция управления. В этом случае можно строить скрытые наземные пункты наведения, обладающие повышенной живучестью.

Также следует отметить, что использование принципа моноимпульсной системы обратного типа не отрицает одновременного использования принципов классической моноимпульсной обработки сигнала, принимаемого приёмниками цифровой РЛС. Значения оценок пеленгов, полученных с помощью прямой и обратной моноимпульсной обработки, можно комплексировать с алгоритмами определения типа помехи. На основании решающего правила, исходя из помеховой обстановки, компенсировать её, переходя к обратному, классическому принципу, либо применять другие алгоритмы защиты от помехи в зависимости от её типа.

Внедрение в действующие образцы ЗРК и ЗРС вышеуказанных методов потребует только перепрограммирования приёмо-передающих модулей.

\section{Список литературы}

1. Родс Д. Р. Введение в моноимпульсную радиолокацию / пер. с англ. под ред. Л. Д. Бахраха. М.: Сов. радио, 1960.159 с. ная радиолокация. М.: Сов. радио, 1970. 392 с. 3. Вексин С. И. Обработка радиолокационных сигналов в доплеровских головках самонаведения. М.: Изд-во МАИ, 2005. 244 с.

4. Сколник М. Справочник по радиолокации. T. 4. Радиолокационные станции и системы / пер. с англ. под ред. М. М. Вейсбейна. М.: Сов. радио, 1978. $375 \mathrm{c}$.

5. Монзинго Р. А., Миллер Т. У. Адаптивные антенные решётки. Введение в теорию. М.: Радио и связь, 1986. 448 с.

6. Вексин С. И. Компенсация помех по боковым лепесткам в доплеровских головках самонаведения // Радиотехника. 2002. № 9. С. 76-86. 7. Козлов С. В., Карпухин В. И., Сергеев В. И. Синтез вариантов структуры радиолокационных измерителей угловых координат с адаптивной пространственной компенсацией помех // Антенны. 2010. № 6. С. 71-76.

8. Григорян Д. С., Абраменков А. В. Теоретические основы построения обратных моноимпульсных радиолокационных систем с повышенной помехоустойчивостью // Вестник Концерна ПВО «Алмаз - Антей». 2014. № 2. C. 68-77.

\section{Поступила 17.07.14}

2. Леонов А.И., Фомичев К.И. Моноимпульс-

Григорян Даниел Сергеевич - кандидат технических наук, профессор кафедры стрельбы и боевой работы на ЗРС и ЗРК средней дальности Военной академии войсковой противовоздушной обороны имени маршала Советского Союза А. М. Василевского, г. Смоленск.

Область научных интересов: цифровые антенные решётки, цифровая обработка сигналов, цифровой спектральный анализ

Лайко Евгений Анатольевич - кандидат технических наук, преподаватель кафедры стрельбы и боевой работы на ЗРС и ЗРК средней дальности Военной академии войсковой противовоздушной обороны имени маршала Советского Союза А. М. Василевского, г. Смоленск.

Область научных интересов: цифровые антенные решётки, цифровая обработка сигналов, цифровой спектральный анализ.

Бушуев Александр Фёдорович - адъюнкт кафедры стрельбы и боевой работы на ЗРС и ЗРК средней дальности Военной академии войсковой противовоздушной обороны имени маршала Советского Союза А. М. Василевского, г. Смоленск.

Область научных интересов: цифровые антенные решётки, цифровая обработка сигналов, цифровой спектральный анализ.

Торбин Сергей Александрович - адъюнкт кафедры стрельбы и боевой работы на ЗРС и ЗРК средней дальности Военной академии войсковой противовоздушной обороны имени маршала Советского Союза А. М. Василевского, г. Смоленск.

Область научных интересов: цифровые антенные решётки, цифровая обработка сигналов, цифровой спектральный анализ. 